\title{
Witnessing the magnetospheric boundary at work in Vela X-1
}

\author{
V. Doroshenko ${ }^{1}$, A. Santangelo ${ }^{1}$, and V. Suleimanov ${ }^{1,2}$ \\ 1 Institut für Astronomie und Astrophysik, Sand 1, 72076 Tübingen, Germany \\ e-mail: doroshv@astro.uni-tuebingen.de \\ 2 Kazan Federal University, Kremlevskaya 18, 420008 Kazan, Russia
}

Received 9 January 2011 / Accepted 20 February 2011

\section{ABSTRACT}

\begin{abstract}
We present an analysis of the Vela X-1 "off-states" based on Suzaku observations taken in June 2008. Defined as states in which the flux suddenly decreases below the instrumental sensitivity, these "off-states" have been interpreted by several authors as the onset of the "propeller regime". For the first time ever, however, we find that the source does not turn off and, although the flux drops by a factor of 20 during the three recorded "off-states", pulsations are still observed. The spectrum and the pulse profiles of the "off-states" are also presented. We discuss our findings in the framework of the "gated accretion" scenario and conclude that most likely the residual flux is due to the accretion of matter leaking through the magnetosphere by means of Kelvin-Helmholz instabilities (KHI).
\end{abstract}

Key words. X-rays: binaries - pulsars: individual: Vela X-1

\section{Introduction}

Vela $\mathrm{X}-1$ is a persistently active high-mass X-ray binary system $(\mathrm{HMXB})$ consisting of a massive neutron star $\left(1.88 M_{\odot}\right.$, Quaintrell et al. 2003) and a B0.5Ib type super giant HD 77581 with mass of $\sim 23 M_{\odot}$ and radius of $\sim 30 R_{\odot}$ (van Kerkwijk et al. 1995). The average X-ray luminosity of the pulsar, $L_{X} \sim$ $4 \times 10^{36} \mathrm{erg} \mathrm{s}^{-1}$, is explained well by the mass-loss rate of the optical companion of $\sim 10^{-6} M_{\odot} \mathrm{yr}^{-1}$ (Nagase et al. 1986), assuming a simple wind model (Castor et al. 1975) and the observed terminal wind velocity of $\sim 1100 \mathrm{~km} \mathrm{~s}^{-1}$ (Watanabe et al. 2006). The neutron star is eclipsed by the optical companion every orbital cycle of $\sim 8.964 \mathrm{~d}$ (van Kerkwijk et al. 1995). The spin period of the neutron star, $P_{\mathrm{s}} \sim 283.5 \mathrm{~s}$ (Rappaport 1975), has remained almost constant since this discovery. The X-ray spectrum of Vela $X-1$ is described well by a cutoff power law (Nagase et al. 1986). Cyclotron resonance scattering features have been reported at $\sim 25$ (Makishima \& Mihara 1992) and $\sim 50-55 \mathrm{keV}$ (Kendziorra et al. 1992), although the feature at $25 \mathrm{keV}$ is still debated (Orlandini 2006).

The source is strongly variable with an average X-ray luminosity of $\sim 4 \times 10^{36} \mathrm{erg} \mathrm{s}^{-1}$ (assuming a distance of $\sim 2 \mathrm{kpc}$, Nagase 1989). Aside from the usual flaring activity similar to the one observed in other wind-accreting pulsars, abrupt "offstates", in which the source becomes undetectable for several pulse periods, and "giant flares" characterized by an increase in the flux up to a factor of 20 have been observed (Kreykenbohm et al. 2008). Both phenomena are not unusual for Vela $X-1$, although probably neither has a periodic nature (Kreykenbohm et al. 2008). The giant flares of Vela $X-1$ have been compared to those seen in super fast X-ray transients (SFXTs, Walter \& Zurita Heras 2007), and may be connected to the clumpy structure of the wind. In this scenario the wind velocity and the density fluctuations are responsible for the luminosity swings: flares would be associated with the accretion of particularly massive wind clumps, whereas "off-states" can be explained with the onset of the centrifugal barrier (Illarionov \& Sunyaev 1975), triggered by a drop in wind density/velocity (Kreykenbohm et al. 2008).

An alternative "gated accretion" scenario to explain the flaring activity of SFXTs was proposed by Grebenev \& Sunyaev (2007) and Bozzo et al. (2008), who suggested that the observed luminosity swings may be associated to the transition between different accretion regimes, i.e. to the different ways the plasma enters the magnetosphere. It is the interaction of the rotating neutron star's magnetosphere with the plasma that mediates the accretion rate. When the magnetospheric boundary is strong, the matter accumulates close to the magnetospheric boundary. If the growth of the magnetospheric instabilities renders the barrier transparent, the accumulated matter can accrete, thereby producing a bright flare. Observational evidence for this scenario was reported by Bozzo et al. (2008) and Grebenev (2010).

In this work we report on the analysis of the June 2008 Suzaku observation of Vela $\mathrm{X}-1$. We focus on the three "offstates" detected in the observation. To our knowledge it is the first time that this type of activity has been observed with an instrument sensitive enough to constrain the flux in the "off-state", to detect pulsations, and to perform the spectral and pulse profile analysis.

We conclude that the accretion still proceeds during the "offstates", although at a much lower rate. We discuss our result in the framework of the "gated accretion" scenario (Burnard et al. 1983; Bozzo et al. 2008) mentioned above. The abrupt decrease in the accretion rate during the "off-states" may then be explained if the magnetospheric boundary becomes stable with respect to Rayleigh-Taylor instability, but some matter still leaks because the magnetosphere is still unstable with respect to Kelvin-Helmholz instabilities (KHI).

\section{Data analysis and results}

The observation we rely on is a $\sim 100 \mathrm{ks}$ long Suzaku observation (ID 403045010), performed on June 17-18, 2008, about $1.6 \mathrm{~d}$ after the eclipse and close to the periastron passage of the 


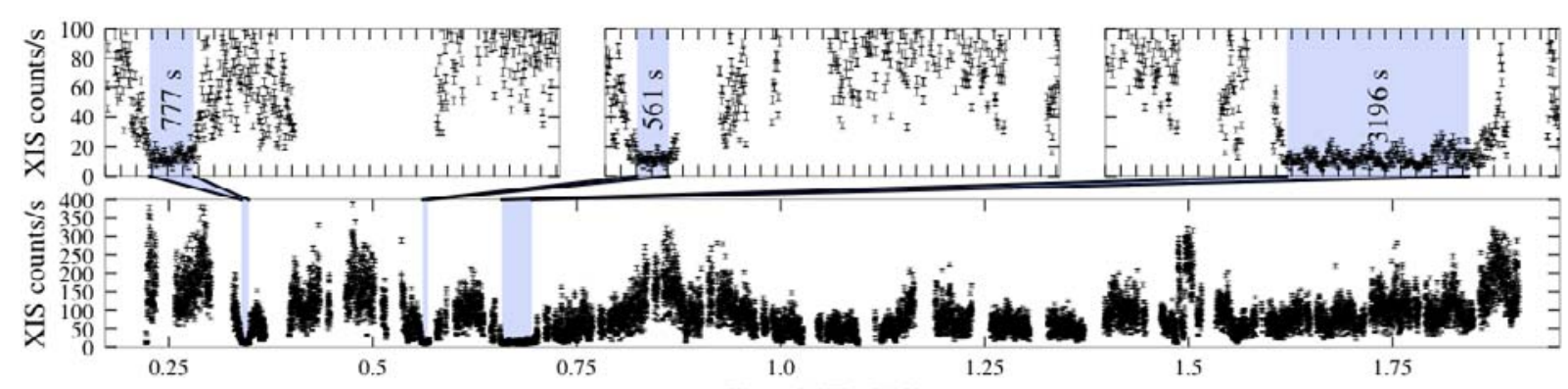

Time. MJD-54634

Fig. 1. Observation-long lightcurve in the $0.4-12 \mathrm{keV}$ energy range using data from all XIS units is shown in the bottom panel. The upper panels show close-up views of the three detected off-states. Here, the time axis is ticked every pulse period.

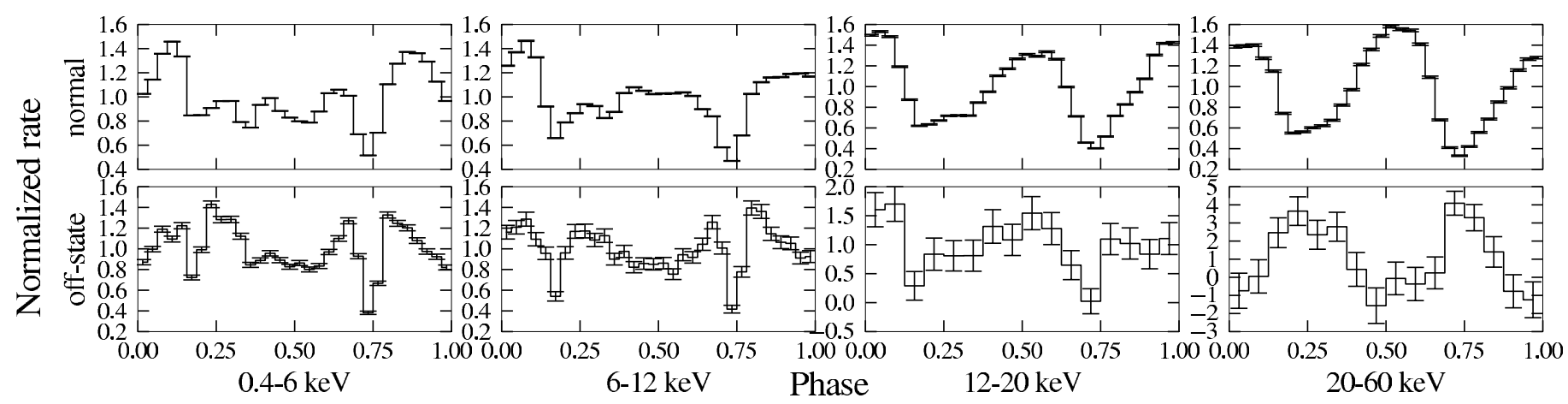

Fig. 2. Pulse profiles in four different energy ranges are shown for the normal (upper panels) and the "off-state" (bottom panels).

source (orbital phase $\sim 0-16$ ). The data was reduced using the HEADAS 6.9 with CALDB version 20100812.

Timing analysis. To improve the quality of the statistics, data from all three XIS units were combined. The lightcurve of the observation in the range $0.4-12 \mathrm{keV}$ is presented in Fig. 1. Three "off-state" episodes, shown in the upper panels of Fig. 1, are observed. As reported in literature (Inoue et al. 1984; Lapshov et al. 1992; Kreykenbohm et al. 1999, 2008), during the "off-states" the sources' flux drops abruptly and recovers after several pulse periods. Marginal evidence of a residual pulsed emission was reported by Inoue et al. (1984) based on Tenma data. With the unprecedented sensitivity of Suzaku not only we do unambiguously confirm these findings but we can also study the "off-states" in detail.

Using the phase-connection technique (Staubert et al. 2009) and assuming the ephemeris by Kreykenbohm et al. (2008), we determined the pulse period to be $P_{\mathrm{s}}=283.473(4)$. All uncertainties quoted are at $1 \sigma$ confidence level unless stated otherwise. The marginal evidence of spin-up is not statistically significant and may be attributed to the uncertainty of the orbital parameters. No change in the pulse period in "off-states" could be measured. Based on the obtained timing solution, we constructed energy-resolved pulse profiles for the entire observation and for the "off-states" by combining all three episodes. As can be seen in Fig. 2, the pulse profiles vary significantly with both energy and luminosity. Very remarkable is the change around phase $\sim 0.75$ at hard energies $(20-60 \mathrm{keV})$ between the normal and the "off-state" profile.

We also investigated the flux distribution of the source following the approach of Fürst et al. (2010), who shows that the flux distribution is approximately lognormal based on INTEGRAL data. Although the lognormal distribution generally describes our data, an excess appears at low count rates (Fig. 3). This excess is due to the "off-states". The flux distribution of the "off-states" is still approximately lognormal (Fig. 3) but differs considerably from the distribution of the rest of the lightcurve. It is the "off-states" component that mostly contributes to the lowcountrate flank seen in the overall flux histogram. Implications of this finding are discussed below.

Spectral analysis. We first analyzed the average spectrum of the entire observation to establish a baseline for the analysis of the "off-state" data. Several phenomenological continua, based on the models reported in literature for Vela $X-1$, were used to fit the average spectrum of the source. None of these models was able to describe the broad-band $0.4-70 \mathrm{keV}$ spectrum. In particular, the spectrum below $5 \mathrm{keV}$ is poorly described by cutoff power-law models.

We used two components to model the continuum, combining a Comptonization model by Titarchuk et al. (1996) and a power law. Photoelectric absorption at lower energies, a number of emission lines, and an iron absorption edge at $\sim 7.26$ (Nagase et al. 1986) were also necessary to fit our data. Two CRSF harmonics were also required by the fit and were modeled using a multiplicative Gaussian profile. The best-fit parameters are summarized in Table 1 . The quality of the statistics is significantly lower for the "off-state" data, so a simpler model was used in this case: an absorbed Comptonization model with the addition of an iron absorption edge. No other spectral features were required by the fit. The results of the best fit are presented in Table 1, and the best-fit spectra and the fit residuals are shown in Fig. 4. The average absorption-corrected flux in the $0.4-70 \mathrm{keV}$ energy range was $\sim 3.8 \times 10^{-9} \mathrm{erg} \mathrm{s}^{-1}$ for the complete observation and $\sim 5 \times 10^{-10} \mathrm{erg} \mathrm{s}^{-1}$ for the "off-state" spectrum. Results of a more detailed spectral analysis, including phase-resolved spectra, will be published elsewhere.

It is interesting to note that the "off-state" spectrum differs considerably from the spectrum observed during the eclipses when it is dominated by emission-lines (Watanabe et al. 2006) 
Table 1. Best-fit parameters of the normal and "off-states" spectra with uncertainties at $1 \sigma$ confidence.

\begin{tabular}{|c|c|c|c|c|c|c|c|c|c|c|c|c|c|}
\hline & $\bar{T} \overline{T_{0, \mathrm{c}}}$ & $k T_{\mathrm{c}}$ & $\tau_{\mathrm{c}}$ & $A_{\text {comp }}$ & $\overline{N_{\mathrm{H}}}$ & $\Gamma$ & $A_{\Gamma}$ & $E_{\mathrm{cyc}, 25}$ & $\sigma_{\text {cyc }, 25}$ & $\tau_{\mathrm{cyc}, 25}$ & $E_{\text {cyc, } 50}$ & $\sigma_{\mathrm{cyc}, 50}$ & $\tau_{\mathrm{cyc}, 50}$ \\
\hline 'On' & $0.98(2)$ & $7.97(3)$ & $15(2)$ & $0.07(2)$ & $1.45(8)$ & $3.2(3)$ & $0.07(2)$ & $26.6(9)$ & $7(1)$ & $0.3(1)$ & $55(3)$ & $13(4)$ & $1.7(1)$ \\
\hline "Off" & $0.83(8)$ & $21.0(1)$ & $7_{-4.4}^{1.5}$ & $\leq 0.03$ & $1.4(1)$ & & & & & & & & \\
\hline
\end{tabular}

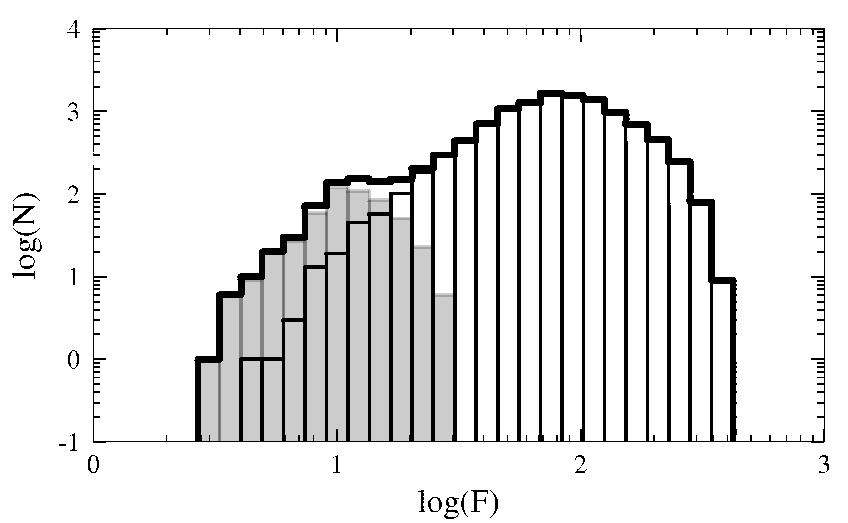

Fig. 3. Histogram of the measured count-rate of the XIS lightcurve (solid line). The same is shown for the off-states (shaded) and for the rest of the lightcurve separately.

originating in the surrounding plasma illuminated by the X-rays emitted by the eclipsed pulsar.

\section{Interpretation and discussion}

First, we would like to summarize the observed properties of the source in its "off-state"

- The flux drops by a factor of ten or more on a timescale comparable to the pulse period. The source remains "off" for several pulse periods, and then the flux is restored to the previous level on the same short timescale.

- Vela X-1 is observed to pulsate in the "off-state". No pulse frequency change has been detected in the "off-state".

- A drastic change in the shape of the pulse profile shape appears at high energies. The narrow dip at pulse phase $\sim 0.75$, seen in the profiles of the normal state at all energy ranges as well as in the "off-state" profiles at lower energies, is substituted by a prominent peak in the "off-state" profile at hard energies (20-60) keV. This leads to a significant increase in the fraction of the pulsed emission in the hard energy range for the "off-states".

- The flux distributions of the normal and "off-states" are significantly different. The overall distribution is composed of two approximately lognormal peaks.

- Although poorly constrained at high energies, the spectrum changes significantly during the "off-state". The temperature of the Comptonizing medium increases, whereas the optical depth decreases. No CRSF is required by the data, although the statistics at high energies does not allow us to rule out the presence of a CRSF completely, especially if the CRSF is shifted to higher energies. The absorption corrected flux in $0.4-70 \mathrm{keV}$ energy range is $\sim 5 \times 10^{-10} \mathrm{erg} \mathrm{cm}^{-2} \mathrm{~s}^{-1}$, which corresponds to a luminosity of $\sim 2.4 \times 10^{35} \mathrm{erg} \mathrm{s}^{-1}$ for a distance of $2 \mathrm{kpc}$.

The observed pulsations, the luminosity and the hard spectrum of the "off-states" can only be explained if the emission is powered by the accretion of plasma onto the magnetized neutron star. The absence of emission lines in the "off-state" spectrum

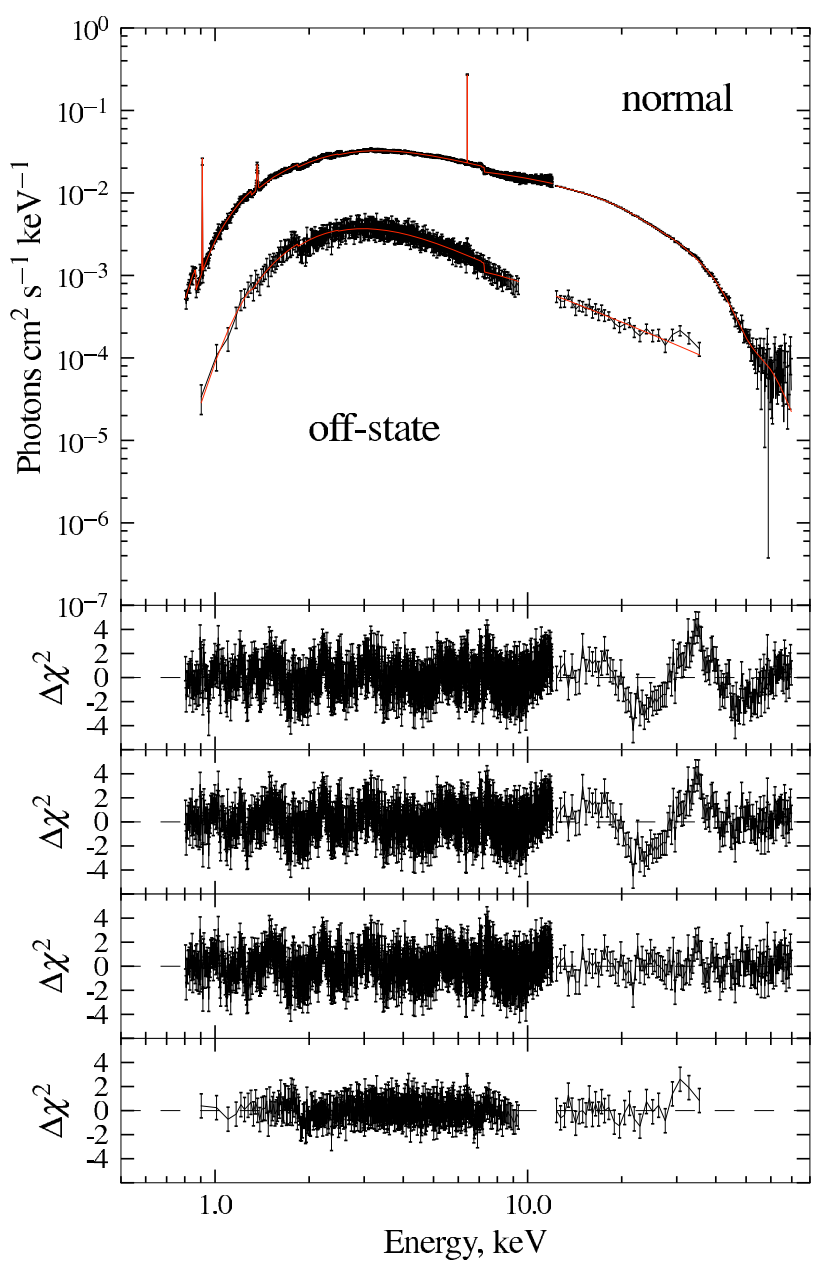

Fig. 4. Unfolded spectrum of the normal and "off states". For the normal spectrum XIS0 and HXD PIN data were used. For the "off" spectrum data from all XIS units were combined to obtain a larger statistics. Best-fit residuals are also shown from top to bottom for: the normal state without CRSF; with the inclusion of a CRSF at $55 \mathrm{keV}$; with the inclusion of two CRSFs, and, eventually, for the "off-state" spectrum.

strongly suggests that the source is not eclipsed, but instead exhibits an intrinsic drop in luminosity, hence in the accretion rate. The timescale of the state transition makes it difficult, as argued by Kreykenbohm et al. (2008), to explain the onset of the "offstates" with a sudden decrease in wind density and/or velocity, and suggests a magnetospheric origin of the state transition.

This agrees with the observed flux distribution. If the "offstates" were due to drops in the wind density, one would expect them to contribute to the lower-flux tail of the normal flux distribution. On the contrary, they form a distinct low-flux peak as observed in Fig. 3. The lognormal flux distribution is most likely caused, as discussed by Fürst et al. (2010), by the "grinding" of a clumpy wind by the magnetosphere, while changes in the distribution parameters may be associated with changes in the way the magnetosphere-plasma interaction proceeds.

As discussed by Burnard et al. (1983), plasma generally enters the magnetosphere of accreting pulsars according to various 
instabilities. These authors also conclude that, for the observed luminosities and spin-periods typical of bright accreting pulsars, the plasma mainly penetrates the magnetospheric boundary via Rayleigh-Taylor instabilities. If the accretion rate decreases, the rotating magnetosphere will inhibit accretion via RayleighTaylor, therefore, for low-luminosity pulsars with intermediate rotation rates, the KHI accretion channel dominates (Burnard et al. 1983).

The ways through which the plasma can penetrate the magnetosphere have been reviewed more recently by Bozzo et al. (2008), who also provide estimates for the leak rates of various mechanisms. For a system with parameters similar to Vela X-1 in "off-state", the highest rate is expected to be provided by KHI (see Sect. 3.2.2 of Bozzo et al. for the details). The accretion luminosity is estimated in this case to be

$$
\begin{aligned}
& L_{\mathrm{KH}} \simeq G M_{\mathrm{NS}} \dot{M}_{\mathrm{KH}} / R_{\mathrm{NS}} \\
& =7.4 \times 10^{35} \eta_{\mathrm{KH}} R_{\mathrm{M} 10}^{3}\left(1+16 R_{\mathrm{G} 10} /\left(5 R_{\mathrm{M} 10}\right)\right)^{3 / 2} \frac{\sqrt{\rho_{\mathrm{i}} / \rho_{\mathrm{e}}}}{1+\rho_{\mathrm{i}} / \rho_{\mathrm{e}}} \mathrm{erg} \mathrm{s}^{-1}
\end{aligned}
$$

here $R_{\mathrm{G} 10}$ and $R_{\mathrm{M} 10}$ are the capture and magnetosphere radius respectively, in units of $10^{10} \mathrm{~cm}$; $\rho_{\mathrm{i}, \mathrm{e}}$ are the densities within and outside of the magnetosphere. According to Bozzo et al. (2008), $\eta_{\mathrm{KH}} \sim 0.1$ and the density ratio is estimated to be between

$$
\frac{\sqrt{\rho_{\mathrm{i}} / \rho_{\mathrm{e}}}}{1+\rho_{\mathrm{i}} / \rho_{\mathrm{e}}}=\left\{\begin{array}{l}
\eta_{\mathrm{KH}} h^{-1} R_{\mathrm{M} 10}^{3 / 2} P_{\mathrm{s} 283.5}^{-1} \\
0.1 \eta_{\mathrm{KH}} h^{-1} R_{\mathrm{M} 10}^{1 / 2} v_{8}
\end{array}\right.
$$

where $h$ is the fractional height of the area where the plasma and the magnetic field coexist, in units of the total thickness of the KHI unstable layer (Burnard et al. 1983), and $P_{\mathrm{s} 283.5}$ is the spinperiod in units of $283.5 \mathrm{~s}$. We assume a canonical neutron star radius of $R_{\mathrm{NS}} \sim 10 \mathrm{~km}$. In the case of Vela X-1, for the observed "off-state" luminosity of $\sim 2.4 \times 10^{35} \mathrm{erg} \mathrm{s}^{-1}$, a magnetic field of $B \geq 2 \times 10^{13} \mathrm{G}$ is required, if the KHI unstable layer is relatively thin $(h \sim 0.05)$, or $B \sim 10^{14} \mathrm{G}$, if $h \sim 1$, as suggested by Burnard et al. (1983).

Evidence of such a high magnetic field in Vela $X-1$, surprisingly stronger than the one estimated from the CRSF energy, are extensively discussed in Doroshenko et al. (2011). Here, we wish only to point out that the discrepancy still can be cleared if the line formation region is located several kilometers above the neutron star surface. In fact, for the average observed luminosity of the normal state, $\sim 4 \times 10^{36} \mathrm{erg} \mathrm{s}^{-1}$, one expects that an accretion column with height up to $\sim 10 \mathrm{~km}$ will arise (Lyubarsky \& Sunyaev 1988; Doroshenko et al. 2010). This implies a factor of ten decrease in field strength at top of the column given that $B \sim B_{0}\left(\left(R_{\mathrm{NS}}+10\right) / R_{\mathrm{NS}}\right)^{-3} \sim 0.1 B_{0}$. It is therefore sufficient to assume that the accretion column exists and that the observed CRSF forms closer to the top of the column to reconcile the strong magnetic field required by the observed "off-states" luminosity and the measured CRSF centroid energy.

The observed change in the high-energy pulse profile is explained under the assumption that an accretion column does indeed exist. The sharp dip, evident at high luminosity and softer energies, would then be due to the eclipse of the polar cap by the accretion column. As the luminosity drops and the column ceases to exist, the hard X-rays can pierce through and the polar cap is observed directly: a pronounced peak is observed instead of a dip. In other words, the high-amplitude peak, which appears in the "off-state" pulse profile at hard energies around pulse phase 0.75 can be attributed to the direct emission from the polar cap. In this scenario, the accretion stream would still absorb the soft X-rays so the dip is still observed at lower energies. A similar scenario is discussed by Klochkov et al. (2008) to explain the pulse profile variations of EXO 2030+375 during outbursts.

\section{Conclusions}

We presented an analysis of the "off-states" of Vela X-1 observed during a $100 \mathrm{ks}$ Suzaku observation of the source. For the first time ever, we have been able to study and characterize the properties of Vela X-1 during the so-called "off-states", detecting pulsations and measuring the spectrum and flux distribution. We also confirm the presence of a CRSF at $25 \mathrm{keV}$ in the normal state of the source. We conclude that these observational results strongly suggest that the emission is still powered by accretion and that the drop in luminosity has a magnetospheric origin. The observed X-ray luminosity of the "off-state" may be naturally explained in the gated accretion scenario originally proposed by Burnard et al. (1983) and recently investigated by (Bozzo et al. 2008), if the neutron star is strongly magnetized and the plasma enters the magnetosphere via Kelvin-Helmholz instabilities. To our knowledge this is the first time that the theoretical considerations of magnetosphere-plasma interactions proposed so far (Burnard et al. 1983; Bozzo et al. 2008) find an observational confirmation. The same interpretation might apply to several other sources for which episodes have been reported that are similar to the "off-states" observed in Vela X-1 (Naik et al. 2005; Göğüş et al. 2011).

Acknowledgements. V.D. and V.S. thank the Deutsches Zentrums für Luftund Raumfahrt (DLR) and Deutsche Forschungsgemeinschaft (DFG) for financial support (grants DLR 50 OR 0702 and SFB/Transregio 7: "Gravitational Wave Astronomy"). V.S. was also supported the Russian Foundation for Basic Research (grant 09-02-97013-p-povolzh'e-a).

\section{References}

Bozzo, E., Falanga, M., \& Stella, L. 2008, ApJ, 683, 1031 Burnard, D. J., Arons, J., \& Lea, S. M. 1983, ApJ, 266, 175 Castor, J. I., Abbott, D. C., \& Klein, R. I. 1975, ApJ, 195, 157 Doroshenko, V., Santangelo, A., Suleimanov, V., et al. 2010, A\&A, 515, A10 Doroshenko, V., Suleimanov, V., \& Santangelo, A. 2011, A\&A, submitted Fürst, F., Kreykenbohm, I., Pottschmidt, K., et al. 2010, A\&A, 519, A37 Göğüş, E., Kreykenbohm, I., \& Belloni, T. M. 2011, A\&A, 525, L6 Grebenev, S. A. 2010, in PoS(extremesky2009)060

Grebenev, S. A., \& Sunyaev, R. A. 2007, Astron. Lett., 33, 149

Illarionov, A. F., \& Sunyaev, R. A. 1975, A\&A, 39, 185

Inoue, H., Ogawara, Y., Waki, I., et al. 1984, PASJ, 36, 709

Kendziorra, E., Mony, B., Kretschmar, P., et al. 1992, in NASA Conf., 3137, 217 Klochkov, D., Santangelo, A., Staubert, R., \& Ferrigno, C. 2008, A\&A, 491, 833 Kreykenbohm, I., Kretschmar, P., Wilms, J., et al. 1999, A\&A, 341, 141 Kreykenbohm, I., Wilms, J., Kretschmar, P., et al. 2008, A\&A, 492, 511 Lapshov, I. Y., Syunyaev, R. A., Chichkov, M. A., et al. 1992, Svt. Ast. L., 18 Lyubarsky, Y. E., \& Sunyaev, R. A. 1988, Pis'ma Astron. Zh., 14, 920 Makishima, K., \& Mihara, T. 1992, Yamada Conference XXVIII Proc. Nagase, F. 1989, PASJ, 41, 1

Nagase, F., Hayakawa, S., Sato, N., Masai, K., \& Inoue, H. 1986, PASJ, 38, 547 Naik, S., Paul, B., \& Callanan, P. J. 2005, ApJ, 618, 866

Orlandini, M. 2006, Adv. Space Res., 38, 2742

Quaintrell, H., Norton, A. J., Ash, T. D. C., et al. 2003, A\&A, 401, 313

Rappaport, S. 1975, IAU Circ., 2869, 2

Staubert, R., Klochkov, D., \& Wilms, J. 2009, A\&A, 500, 883

Titarchuk, L., Mastichiadis, A., \& Kylafis, N. D. 1996, A\&AS, 120, C171 van Kerkwijk, M. H., van Paradijs, J., Zuiderwijk, E. J., et al. 1995, A\&A, 303 Walter, R., \& Zurita Heras, J. 2007, A\&A, 476, 335

Watanabe, S., Sako, M., Ishida, M., et al. 2006, ApJ, 651, 421 